\title{
JCCS-A journal for translational research
}

\author{
Andrew Leask $\cdot$ Bernard Perbal
}

Received: 13 July 2010 / Accepted: 14 July 2010 /Published online: 31 July 2010

(C) The International CCN Society 2010

It has now been 3 years since the inaugural issue of the Journal of Cell Communication and Signaling as published by Springer. Taking into consideration its predecessor, Cell Communication and Signaling (JCCS) published by Biomed Central, it has now been 7 years since the International CCN Society has sponsored a journal. The original intent of the International CCN Society was to create a journal disseminating knowledge concerning the $\mathrm{CCN}$ family of matricellular proteins.

In 2003, it was perceived that the $\mathrm{CCN}$ family of proteins was under-recognized within the broader scientific community and was in need of a journal emphasizing their role. It is our belief that, in this regard, the journal has been a great success and has aided in the overall growth of interest in the CCN family as critical modifiers of signaling pathways involved with a wide range of physiological activities. In particular, we feel that the CCN family is now recognized by the translational research community as playing an essential role in human diseases including cancer and fibrosis by modifying cellular responses to signaling molecules including cytokines and extracellular matrix. Indeed, we feel that detailed knowledge of these interactions will be essential, in the future, for developing novel therapeutic approaches to combat disease.
In 2010, we recognize that there is a need for a journal that aims to publish quality manuscripts focusing on the complex, context-dependent interactions between cells and their environment and the role of these interactions in disease. We have decided to alter and broaden the focus of JCCS to fill this gap.

Thus, with this issue of JCCS, we are instituting several changes. First, in consultation with Springer, we have modernized the cover of JCCS, which will now change each quarter to include a photograph or illustration of a key concept published in the issue. Second, the membership of the editorial board has been altered to reflect the broader focus of the journal on translational research. Finally, to emphasize the continuity with the original scope of JCCS, Prof Bernard Perbal will remain as Executive Editor, but Prof Andrew Leask will now assume a new position as Scientific Editor.

We hope that you will consider sending articles to JCCS. We continue to promise a rapid and fair review process focused on the quality of the science presented.

Sincerely

Andrew Leask

Bernard Perbal

\footnotetext{
A. Leask $(\bowtie)$

Schulich School of Medicine and Dentistry,

University of Western Ontario,

London, Canada

e-mail: Andrew.Leask@schulich.uwo.ca

B. Perbal

e-mail: bperbal@gmail.com
} 